\title{
Immuno-Oncology Medicines: Policy Implications and Economic Considerations
}

\author{
Georges Adunlin, PhD; Jenny Dong, PharmD; Maisha Kelly Freeman, PharmD, MS, BCPS, FASCP
}

Samford University

\begin{abstract}
Significant progress has taken place in the field of cancer immunotherapy in recent years. Cancer immunotherapy, particularly immune checkpoint inhibitors, have shown rather dramatic results and are believed to have completely transformed the field of oncology. However, these transformational therapies are more expensive than previous cancer therapies. As more cancer immunotherapy agents are being developed, with some already being marketed, it is important to consider how economic constraints will shape health policy and value assessment related to these agents. A number of strategies have been suggested to alleviate the price burden and the ensuing concerns about the sustainability of publicly funded healthcare systems. Among these strategies, value-based pricing (VBP) for innovative drugs dominates the headlines in the field of oncology. The specifics of how VBP may be implemented in the United States is still unclear. Nonetheless, policy reform and economic considerations will have to be incorporated into the planning of VBP. The objective of this paper is multifold: (i) to identify the factors affecting the impact of cancer immunotherapy on healthcare cost; (ii) to critically appraise current approaches used to assess the value of novel cancer therapies; (iii) to assess the methodological challenges associated with the economic evaluation of cancer immunotherapy. As the health care system in the U.S transitions toward a valuebased model, the need for a formal value assessment framework is warranted in cancer immunotherapy.
\end{abstract}

Keywords: cancer, immunotherapy, value-based pricing, immune checkpoint inhibitors

\section{Introduction}

Cancer causes significant morbidity and mortality in the United States (U.S.) and imposes a substantial economic burden on individuals and society as a whole. ${ }^{1-3}$ Despite therapeutic advances in chemotherapy and targeted therapy, the prognosis of most metastatic solid tumors remains poor, as they are typically incurable. This phenomenon accentuates the need for new therapeutic approaches. ${ }^{4}$ Immunotherapy, commonly defined as treatment of disease by inducing, enhancing, or suppressing an immune response, has become the mainstay of pharmaceutical innovation. ${ }^{5,6}$ Immunooncology (I-O) is the area of cancer research that focuses on understanding the interaction of the immune system with cancer cells and discovers ways to use or enhance the patient's own immune system to stop the growth of cancer cells. ${ }^{7,8}$ The concept of I-O dates back to the $18^{\text {th }}$ century when William B. Coley injected streptococcal organisms into a patient with inoperable cancer. ${ }^{9,10}$ Dr. Coley's injection, which became known as Coley's toxins, resulted in shrinkage of the tumor. ${ }^{11}$

The emergence of I-O agents is changing the landscape of the management of metastatic stage IV cancer, offering potential for long-term survival and improved quality of life. ${ }^{5,6}$ The main types of immunotherapy presently used to treat cancer include monoclonal antibodies, immune checkpoint inhibitors

Corresponding author: Georges Adunlin, PhD

Assistant Professor

Dept of Pharmaceutical, Social \& Admin Sciences McWhorter School of Pharmacy Samford University

Email: gadunlin@samford.edu
(ICls), therapeutic cancer vaccines, and other non-specific immunotherapies. ${ }^{12,13}$ Tumor-specific monoclonal antibodies can elicit a direct or indirect immune response that leads to cell death. There is a variety of monoclonal antibodies that work via different mechanisms of action to cause cell death. ${ }^{12,13}$ Immune checkpoints refer to a group of molecules on the surface of both self-cells and cells of the immune system that send a coinhibitory stimulus that attenuates an immune response. ICls overcome negative regulatory mechanisms utilized by tumor cells to evade the immune system. Therapeutic cancer vaccines are designed to stimulate the patient's own immune system against tumor antigens. ${ }^{12,13}$ Non-specific immunotherapies stimulate the immune system in a general way to increase activity against cancer cells. ${ }^{12,13}$ Some examples include manmade versions of cytokines, a chemical in immune cells, such as interleukins and interferons. ${ }^{12,13}$ Checkpoint inhibitors are the most rapidly evolving class of immunotherapy drugs and provide the most extensive data for discussion. The cost of I-O drugs is remarkably higher compared to standard-of-care, with an average price ranging between $\$ 120,000$ to $\$ 150,000$ for a year of treatment in the U.S. ${ }^{14,15}$ The high price of novel cancer agents is placing increasing pressure on limited healthcare budgets. Therefore, the economic impact of the development and use of I-O drugs is an important consideration for pricing and reimbursement policies. This paper outlines general principles of economic evaluations and health-related policies for cancer immunotherapy, with a focus on checkpoint inhibitors. The objective of this paper is multifold: (i) to identify the factors affecting the impact of cancer immunotherapy on healthcare cost; (ii) to critically appraise current approaches used to assess the value of novel cancer therapies; (iii) to assess the methodological challenges associated with the economic evaluation of cancer immunotherapy. 


\section{Health Policy in the Era of Cancer Immunotherapy}

According to an IMS Health's Global Oncology Trend Report, worldwide spending on oncology medications has grown to $\$ 113$ billion in 2016, an increase from $\$ 91$ billion five years earlier. ${ }^{16}$ The U.S represents the largest market for oncology drugs accounting for approximately $46 \%$ of total spending. ${ }^{16}$ Spending has been mostly attributed to the rising cost of cancer drugs.,17 The average per capita price of cancer drugs for one year of therapy in the U.S. has increased from $\$ 5,000-$ $\$ 10,000$ before the year 2000 to more than $\$ 100,000$ by 2012 . However, little correlation between the actual efficacy of the new drugs and their prices has been elucidated. ${ }^{18} \mathrm{I}$-O agents are clearly at the forefront of the debate about drug pricing since the approval of diverse agents in recent years and with more products in clinical development. The price for one month of treatment at initial FDA approval was $\$ 39,947$ for ipilimumab (for a Progression Free Survival (PFS) of 2.9 months), $\$ 8,725$ for pembrolizumab (for an Overall Response Rate (ORR) of $26 \%$ ), and $\$ 12,500$ for nivolumab (for a PFS of 6.9 months). The global immunotherapy drugs market is large and is projected to reach $\$ 201.52$ billion by 2021 from $\$ 108.41$ billion in 2016. ${ }^{19}$

Many countries have adopted a unique mechanism in an attempt to control pharmaceutical expenditures, ensure value for money and sustainability, and facilitate patient access. This mechanism consists of health technology assessment (HTA) programs that consider clinical safety and efficacy, price, and overall value before a recommendation for reimbursement is made for a new cancer drug. These programs are established through agencies in countries including Britain (National Institute for Health and Care Excellence [NICE]), Australia (Pharmaceutical Benefits Advisory Committee [PBAC]), Sweden (Swedish Council on Technology Assessment in Health Care [SBU]), the Netherlands (Dutch Healthcare Insurance Board/College voor Zorgverzekeringen [CVZ]), and Germany (Institute for Quality and Efficiency in Health Care [IQWIG]). In Canada, oncology drugs are reviewed by a separate entity, the pan-Canadian Oncology Drug Review (pCODR). In contrast to other developed countries, the U.S does not have a formalized HTA system. ${ }^{20,21}$

The uncertainty that might arise with coverage decision of I-O agents could be well illustrated with the case of Sipuleucel-T, an autologous cellular immunotherapy for the treatment of asymptomatic or minimally symptomatic metastatic castrateresistant prostate cancer, which costs approximately $\$ 93,000$ for a typical three-course cycle. ${ }^{22}$ Sipuleucel-T was FDA approved in 2010 after a phase 3 trial demonstrated a median overall survival (OS) benefit of 4 months compared to the control group. After FDA approval, the U.S Centers for Medicare \& Medicaid Services (CMS) made the unusual move to launch a National Coverage Analysis to review the labeled use of Sipuleucel-T to determine whether it improved health outcomes and if it should still be covered for the Medicare population. On March 30, 2011, the CMS announced their final national coverage determination for Sipuleucel-T and indicated that the drug improved health outcomes for its beneficiaries. ${ }^{23}$ In the UK, NICE has not recommended Sipuleucel-T for treating adults who have asymptomatic or minimally symptomatic metastatic non-visceral hormonerelapsed prostate cancer for which chemotherapy is not yet clinically indicated because the available evidence determined that the price the National Health Service (NHS) is required to pay for the drug is too high for the benefit it may provide to patients. ${ }^{24}$ The NICE evaluation Committee indicated that the cost per quality-adjusted life year (QALY) for Sipuleucel-T was well above the threshold range usually considered a costeffective use of UK NHS resources (i.e., $£ 20,000-£ 30,000$ per QALY gained) and that Sipuleucel-T did not meet the criteria for end-of-life consideration. The controversy about the coverage decision of Sipuleucel-T opens a new avenue for Medicare decisions and future coverage.

Although regulations in the U.S. concerning cancer drugs faces many challenges, the pricing policy has doubtlessly improved over the last two decades. Nevertheless, some important factors should be addressed in the future policy agenda. ${ }^{25}$ The American Association of Clinical Oncology (ASCO) has done a very good job at highlighting some of these factors in a 2015 report. Specifically, ASCO has put forth a multiple innovative payment and care delivery models recommending that: (a) CMS expands its efforts to pilot alternative payment models beyond its Oncology Care Model; (b) Congress provides a fair, adequate and stable payment environment for oncology practice; (c) Private insurers partner with CMS, patients, and providers to test promising new payment and care delivery models; (d) Oncology professionals engage in testing/evaluating new payment and care delivery models.

\section{Economic Value of Immuno-Oncology Therapies}

"Value represents a composite measure of drug utility consisting of clinical, economic and quality of life $(\mathrm{QOL})$ related attributes." ${ }^{26}$ The QALY as part of the incremental costeffectiveness ratio (ICER) combines these three attributes into a single measure that puts a figure on the benefits of most health-care interventions. The QALY is currently recommended as reference case by various HTA agencies. The cost-per-QALY threshold varies substantially by country because not only does gross domestic product (GDP) vary by country, but guidelines for conducting pharmacoeconomics research also differ depending on the country. ${ }^{27}$ In the UK, NICE uses a threshold in the range of $£ 20,000$ to $£ 30,000$, and above a most plausible ICER of $£ 30,000$ per QALY gained. ${ }^{28}$ In Australia, there is no specific threshold for approval; however, between 1994 and 2003, the highest cost per QALY at which a drug was recommended for listing was Australian $\$ 52,400 .^{29}$ Above this value, 9 of 11 applications were rejected or withdrawn by the manufacturer and two were given only conditional approval (subject to price reductions)..$^{29}$ The 
Netherlands has no formal threshold, yet a cutoff value at $€ 20,000$ per QALY is often cited in various reports. ${ }^{30,31}$ In the literature, as well as many other jurisdictions, the commonly used threshold is $\$ 50,000$ cost per QALY gained. ${ }^{31,32}$

The World Health Organization (WHO) considers a drug to be cost-effective if its threshold value is within one to three times the local GDP per capita per QALY gained. ${ }^{33}$ However, the rationality of this suggested threshold draws controversy..$^{31,34}$ For illustration purposes, using a high-income country like the U.S. (i.e., per capita GDP $=\$ 54,629.50$ ), the three-time threshold for cost-effectiveness would be roughly $\$ 163,888.50$ per QALY gained. For an upper-middle-income country such as Mexico (per capita GDP $=\$ 10,230.20$ ), the cost per QALY gained threshold would be U.S $\$ 30,690.60$ (The World Bank, 2015). For a lower-middle-income country such as Indonesia (per capita GDP $=\$ 3,491.90$ ), the cost per QALY gained threshold would be $\$ 10,475.70$. In contrast, the threshold for economic value for a low-income country, such as Liberia (per capita GDP $=\$ 461.00$ ) would be $\$ 1,383$ per QALY gained (The World Bank, 2015). Based on this logic, the price of the same drug would considerably vary from one country to another. While the cost per QALY of many new cancer drugs is contentious to estimate, they often exceed the threshold of $\$ 100,000$ per QALY gained.

Critics have expressed concerns over the ethics and equity associated with valuing health gains in terms of QALYs and the appropriateness of its use in health care decision-making. 35,36 In fact, QALY may discriminate between individuals on age grounds since younger individuals are more likely to be healthier while the elderly are more prone to sickness. ${ }^{37}$ In recent years, many countries have taken a stance on QALY thresholds. In the UK, NICE has proposed to no longer use the cost-per-QALY information to make recommendations, but rather rely on cost-effectiveness assessments to provide input into price negotiations for new technologies. ${ }^{38}$ In Germany, the IQWIG implemented a new system for estimating the value of medical technologies but rejected the cost-per-QALY model on ethical and methodological grounds. ${ }^{39}$ In cases of uncertainty about the relative effectiveness, payers are turning to novel concepts such as value-based pricing (VBP).

\section{Value-based pricing}

Value-based health care has moved to the forefront of the health policy agenda, as public payers search for an approach to improve outcomes while containing cost. ${ }^{40}$ However, the U.S. has yet to learn from the experiences of other industrialized countries by including the concepts of costeffectiveness (CEA) and VBP as part of reimbursement decision-making, which may lead to improved outcomes for patients. Value-based pricing consists of negotiating prices for new pharmaceuticals based on the value the new drug offers society, as assessed through HTA. ${ }^{41,42}$ In the UK, there is already a version of VBP in place for new cancer medicines, which defines their maximum acceptable NHS prices. ${ }^{43,44}$ In the U.S., the government does not provide adequate leverage in negotiating drug prices. Medicare, the government's largest health plan does not directly negotiate with drug manufacturers over prices for prescription drugs covered under the Part $B$ benefit or the oral anticancer drugs covered under Medicare's pharmacy “Part D" benefit. ${ }^{45,46}$

Consequently, as effective and more expensive ICls are introduced to the U.S. market, Medicare is left with no other alternative than to pay for these costly drugs. Countries whose law favors the active role of government in pharmaceutical price negotiations have resorted to the VBP concept to reduce pharmaceutical expenditures. ${ }^{47}$

Several value frameworks have been developed to assess the value of cancer drugs, including those by the Institute for Clinical and Economic Review (ICER), the American Society of Clinical Oncology (ASCO), the National Comprehensive Cancer Network (NCCN), and the Memorial Sloan Kettering (MSK) cancer center DrugAbacus. The ICER Value Assessment Framework offers a source of information that can act as the backbone of discussions between insurers, providers, patients, and policymakers regarding matters related to pricing, payment, and the use of new drugs. ICER's Value Assessment Framework separates value into two components: "care value" and "provisional health system value." ${ }^{\text {"8,49 }}$ Care value assessment takes into account comparative clinical effectiveness, incremental costs per outcomes achieved other benefits or disadvantages, and contextual considerations such as ethical and legal concerns that shape the relative priority of the intervention. Provisional health system value is assessed based on whether medication could treat a population with reasonable long-term value and considers the short-term budget impact that would not lead to excessive health care expenditure.

The ASCO value framework developed guidelines to help clinicians, patients, and families through effective shared decision-making to best evaluate treatment options for cancer. ${ }^{50}$ This framework seeks to ensure that intervention costs result directly in beneficial impacts for patients.

The NCCN Evidence Blocks have been devised to ease discussions between physicians and patients for elements of informed decision-making. ${ }^{51}$ It is presented as a visual representation of five value measures that provide important information about specific recommendations found in the NCCN Guidelines including efficacy, safety, quality of evidence, consistency of evidence, and affordability. ${ }^{51}$

MSK's DrugAbacus is a publicly available online tool that allows users to easily calculate the price for a cancer drug based on their chosen settings for its value. ${ }^{52}$ DrugAbacus measures value according to six components: the value of a life year, toxicity, treatment novelty, research and 
development costs, the rarity of the disease it targets, and the population health burden the targeted disease causes.

The need to define value in cancer therapy stems from the continuous growth of both healthcare demand and consumption. The implementation of a VBP scheme in the U.S offers an opportunity to provide patients timely access to drugs, and control drug prices. However, before such a scheme can be implemented, some policies may need to be revised to allow public programs to take advantage of diverse systematic evaluation methods to assess the value of new drugs and to negotiate directly with pharmaceutical manufacturers on price based on their assessment findings. It is also worth underlining that even though the introduction of VBP would safeguard access to effective and innovative IO agents by setting a price that reflects the utility created, it can deter innovation. The VBP scheme is also a single component of many moving parts and this system alone will not address all the concerns over the costs for cancer medications.

\section{Reimbursement and Patient Access}

In an era of unsustainable pricing and the difficulties to define the value of I-O drugs, reimbursement challenges may arise. The clinical and/or economic value attributable to I-O drugs is not well established at the moment. There is no evidence to support whether existing HTA framework and reimbursement processes are appropriate for the new generation of I-O drugs. Therefore, there may be consequences in making unsustainable reimbursement decisions. Instead of the current fee-for-service system, ${ }^{53}$ an improved reimbursement system adapted to novel cancer therapies is warranted. Ideally, under such reimbursement, the cost of I-O agents would be negotiated based on the value that they provide to patients. Patient access to costly new cancer drugs is of great interest and concern to all stakeholders including patients, physicians, payers, manufacturers, and policymakers, who are seeking to limit coverage to subpopulation most likely to benefit from these drugs. ${ }^{54}$

The U.S has a comprehensive mechanism whereby important new drugs become available to American consumers faster than the existing standard and priority review programs for certain conditions including cancer. ${ }^{55}$ The national "Moonshot" initiative to cure cancer initiative is expected to streamline bureaucracy so patients can have easy access to new drugs such as promising new drug combinations. ${ }^{56}$ Yet, there are structural barriers that may prevent patient access to I-O drugs. ${ }^{57}$ When considering strategies to improve access, one must consider the effectiveness of clinical trials in providing sound evidence translatable to broad patient populations. This places an emphasis on trial designs that value inclusivity and use appropriate surrogates as well as predictive markers. Trials are also needed to compare the efficacies of competitor products and utilize "adaptive licensing" plans.
Once therapies are introduced into practice, evidence should subsequently be collected, evaluated, and made available to constituencies responsible for delivery and reimbursement. Policies should promote greater understanding of the potential benefits of I-O drugs and illustrate how they differ from other cancer therapies. Outcomes-based agreements between pharmaceutical and insurance companies such as value-based payments and risk-sharing agreements should be incentivized. These outcome-based agreements would seek to address what really matters to patients, which is long-term quality survival and safety of therapies with the goal of ultimately improving patient access. Medicare should be given the mandate to negotiate prices for high-cost drugs with pharmaceutical manufacturers, which would likely lead to significant overall cost savings. Furthermore, competition should be encouraged to leverage price negotiation, and funding for innovative, socially responsible research on I-O agents should be increased.

\section{Considerations for Economic Evaluation}

As new health care strategies compete with existing ones for limited resources in environments with limited resources, all levels of the health care system are relying on economic evaluations to achieve maximum value for money and to help inform decision making. ${ }^{58,59}$ In many countries, Pharmacoeconomic Evaluations (PEs) plays an increasingly important role in informing clinical development and market access decisions of new innovative medicines. ${ }^{60,61}$ Since there is a lack of information on whether new considerations are to be included in the PE of I-O drugs, a search of the literature was performed to identify all peer-reviewed published articles on PEs of marketed ICI therapies (ipilimumab, pembrolizumab, and nivolumab) and comment on the modes of evaluation. The literature search identified only one cost-effectiveness study of ipilimumab. ${ }^{62}$ There are several factors which may explain the scarcity of PE literature on ICl. First, International health technology assessment HTA agencies often do not publish in the peer-reviewed literature or publish sometime after the evaluation is complete. Economic evaluations of ICls will therefore likely be in the grey literature. Second, ICls may have not been studied extensively, partly due to their novelty.

Due to recent emergence, there are several challenges looming with the PE of ICls, mainly related to methodological issues. The mechanism of action of ICls tends to result in delayed clinical effect. ${ }^{63}$ The clinical experience of ipilimumab has shown that complete response (CR), partial response (PR), or stable disease status can still be achieved after an initial increase in the overall tumor burden. ${ }^{64,65}$ The unique characteristics of ICls also produce long-term survival, resulting in a delay in separation of Kaplan-Meier (KM) survival curves between the control and treatment arms of clinical trials. ${ }^{66-68}$ For instance, KM curves of OS in patients treated with ipilimumab show that survival consistently reaches a plateau at around 2 to 3 years, $, 6,69,70$ as demonstrated in both 
randomized phase 3 trials of ipilimumab in metastatic melanoma. Beyond this time point, the long "tail" of the survival curve reflects the emergence of long-term survivors. Data models for delayed effects of treatment on time-to-event endpoints such as survival violate the proportional hazards assumption. ${ }^{71}$ The proportional hazards assumption stipulates that the survival curves for two strata must have hazard functions that are proportional over time (i.e. constant relative hazard). In other terms, the ratio of hazards is constant and does not depend on time. The unique characteristics of the long-term survival and delayed clinical effect in melanoma constitute a challenge to PE. The point is that the KM curve assumption of proportional hazards (which is violated) and long-term survivors do not apply to standard analytical approaches for OS extrapolation beyond the study follow-up time of the clinical trial. Nonetheless, OS will vary depending on the clinical indication that is being treated and the 'delayed effect' observed for melanoma may not be seen with other cancers. Similarly, not all survival curves for all clinical indications that receive ICls will violate the proportional hazards assumption. Therefore, economic evaluation of $\mathrm{ICls}$ will require caution and in many cases will require the utilization of innovative analytical tools. Several methods have been proposed to overcome some of these challenges. Regarding the delayed clinical effect, in their CEA study of ipilimumab, Barzey et al. indicated that the best fitting parametric curve (lognormal) did not adequately represent the survival of ipilimumab patients. ${ }^{62}$ Therefore, their analysis used an alternative, non-parametric method, which assumed that patients died at a constant rate. Chen proposed the weighted log-rank test as an exploratory analysis to account for the delayed separation of KM curves. ${ }^{72}$ Using the experience of ipilimumab, Annemans and colleagues explored two approaches. ${ }^{73}$ The first method involved a "selected hazard rate" approach which reflected clinical prognosis. The second method was a "broken curve" approach, which found the best mathematical fit of standard survival models within subsets of available data, combined with the use of external epidemiology data. Their investigation then used the Akaike's Information Criterion (AIC), ${ }^{74}$ a measure of the relative quality of statistical models, to determine the best-fit curve.

Due to relative novelty, RCT evidence for ICls should be substantiated with observational studies. Ongoing developments in data and analytical techniques, such as data linkage and propensity score methods, and instrumental variables offer a promising future for observational studies. ${ }^{75,76}$ Economic evaluation requires an appropriate time horizon to fully capture the costs and benefits between the alternative being compared during and beyond the trial. However, RCTs are often characterized by their short time horizon. The extent to which the findings of a clinical trial can be reliably extrapolated from the subjects who participated in the trial to a broader patient population and a range of clinical settings is questionable. Well-designed observational studies can be a reliable tool for addressing the issue of time horizon and generalizing the results of RCTs. In observational studies, individuals are followed for an extended period and are more similar to real-life patients. One of the drawbacks of observational studies is their limited applicability for decisionmaking purposes because the data are usually available after regulatory approval and reimbursement.

High-quality economic evaluation plays a major role in decision making. Understanding and quantifying the uncertainty in an economic evaluation model is paramount to enable decision-makers to correctly interpret the results for specific outcomes, determine whether those results are robust and acceptable to approve an 10 agent for use. Quantification of decision uncertainty in economic evaluation may be improved through the application of value of information methods, and conducting of probabilistic sensitivity analysis.

\section{Conclusion}

With health care costs rising, payers are seeking strategies to contain spending in all areas including prescription drugs. I-O drugs have the potential to revolutionize pharmacotherapy of tumor metastasis and cancer. However, due to their high costs, I-O may also revolutionize spending on prescription drugs. Because of this confluence, stakeholders including patients, physicians, policy-makers, and pharmaceutical companies are grappling with new approaches to price setting and drug value assessment. $1-0$, like most novel cancer therapies, is complex. Thus there is a need to develop appropriate and robust economic evaluation methods to provide a basis for decision-making. Appropriate HTA of I-O drugs is critical to demonstrate their benefit, quantify their potential value, and guide reimbursement decisions.

Conflicts of Interest: We declare no conflicts of interest or financial interests that the authors or members of their immediate families have in any product or service discussed in the manuscript, including grants (pending or received), employment, gifts, stock holdings or options, honoraria, consultancies, expert testimony, patents and royalties.

Funding: The author(s) received no financial support for the research, authorship, and/or publication of this article. 


\section{References}

1. American Cancer Society. Cancer Facts \& Figures 2019. Atlanta: American Cancer Society;2019.

2. Yabroff KR, Dowling EC, Guy Jr GP, et al. Financial hardship associated with cancer in the United States: findings from a population-based sample of adult cancer survivors. Journal of Clinical Oncology. 2016;34(3):259.

3. Mariotto AB, Yabroff KR, Shao Y, Feuer EJ, Brown ML. Projections of the cost of cancer care in the United States: 2010-2020. Journal of the National Cancer Institute. 2011;103(2):117-128.

4. Masters GA, Krilov L, Bailey HH, et al. Clinical cancer advances 2015: Annual report on progress against cancer from the American Society of Clinical Oncology. Journal of clinical oncology : official journal of the American Society of Clinical Oncology. 2015;33(7):786-809.

5. Mellman I, Coukos G, Dranoff G. Cancer immunotherapy comes of age. Nature. 2011;480(7378):480-489.

6. Scagliotti GV, Bironzo P, Vansteenkiste JF. Addressing the unmet need in lung cancer: The potential of immuno-oncology. Cancer treatment reviews. 2015.

7. Blattman JN, Greenberg PD. Cancer immunotherapy: a treatment for the masses. Science (New York, NY). 2004;305(5681):200-205.

8. Rosenberg SA, Yang JC, Restifo NP. Cancer immunotherapy: moving beyond current vaccines. Nature medicine. 2004;10(9):909-915.

9. Coley WB. The treatment of malignat tumors by repeated inoculations of erysipelas: with a report of ten original cases. The American Journal of the Medical Sciences. 1893;105(5):487-510.

10. Coley WB. The therapeutic value of the mixed toxins of the streptococcus of erysipelas and bacillus prodigiosus in the treatement of inoper-able malignant tumors: with a report of One hundred and sixty cases. The American Journal of the Medical Sciences. 1896;112(3):251-280.

11. McCarthy EF. The toxins of William B. Coley and the treatment of bone and soft-tissue sarcomas. The lowa orthopaedic journal. 2006;26:154-158.

12. Curiel TJ. Cancer Immunotherapy: Paradigms, Practice and Promise. Springer Science \& Business Media; 2012.

13. Prendergast GC, Jaffee EM. Cancer immunotherapy: Immune suppression and tumor growth. Academic Press; 2013.

14. Dranitsaris G, Zhu X, Adunlin G, Vincent MD. Cost effectiveness vs. affordability in the age of immunooncology cancer drugs. Expert review of pharmacoeconomics \& outcomes research. 2018:1-7.

15. Incollingo BF. Considering Cost: What's an immunoteraphy Worth? In:2015.
16. Quintiles IMSI. Global Oncology Trends 2017: Advances, Complexity and Cost. 2017.

17. Philipson T, Eber M, Lakdawalla DN, Corral M, Conti R, Goldman DP. An analysis of whether higher health care spending in the United States versus Europe is 'worth it' in the case of cancer. Health affairs (Project Hope). 2012;31(4):667-675.

18. Kantarjian H, Rajkumar SV. Why Are Cancer Drugs So Expensive in the United States, and What Are the Solutions? Paper presented at: Mayo Clinic Proceedings; 2015.

19. Grand View R. Cancer Immunotherapy Market Analysis By Product (Monoclonal Antibodies, Immunomodulators, Oncolytic Viral Therapies, Cancer Vaccines), By Cancer Type, And Segment Forecasts, 2014 - 2025. In. Vol 20172017.

20. Trudeau ME, Chambers A, Christiansen K, Mai H. PanCanadian Oncology Drug Review (pCODR): A unique model to support harmonization of cancer drug funding decisions in Canada. In: American Society of Clinical Oncology; 2018.

21. Rocchi A, Chabot I, Glennie J. Evolution of health technology assessment: best practices of the panCanadian Oncology Drug Review. ClinicoEconomics and outcomes research: CEOR. 2015;7:287.

22. Brower V. Approval of provenge seen as first step for cancer treatment vaccines. Journal of the National Cancer Institute. 2010;102(15):1108-1110.

23. Centers for M, Medicaid S. Proposed decision memo for autologous cellular immunotherapy treatment of metastatic prostate cancer (CAG-00422N). In: March; 2010.

24. National Institute for Health Care and Excellence. Final appraisal determination Sipuleucel-T for treating asymptomatic or minimally symptomatic metastatic hormone-relapsed prostate cancer. NICE technology appraisal guidance [TA332] (2015).

25. Burstein HJ, Griggs JJ, Prestrud AA, Temin S. American society of clinical oncology clinical practice guideline update on adjuvant endocrine therapy for women with hormone receptor-positive breast cancer. Journal of oncology practice. 2010;6(5):243-246.

26. Dranitsaris G, Truter I, Lubbe MS, Amir E, Evans W. Advances in cancer therapeutics and patient access to new drugs. PharmacoEconomics. 2011;29(3):213-224.

27. Dranitsaris G, Papadopoulos G. Health Technology Assessment of Cancer Drugs in Canada, the United Kingdom and Australia: Should the United States Take Notice? Applied health economics and health policy. 2014:1-12.

28. Wonderling D, Sawyer L, Fenu E, Lovibond K, Laramée $P$. National clinical guideline centre cost-effectiveness assessment for the national institute for health and clinical excellence. Annals of Internal Medicine. 2011;154(11):758-765. 
29. Henry DA, Hill SR, Harris A. Drug prices and value for money: the Australian Pharmaceutical Benefits Scheme. Jama. 2005;294(20):2630-2632.

30. Boersma C, Broere A, Postma MJ. Quantification of the Potential Impact of Cost-effectiveness Thresholds on Dutch Drug Expenditures Using Retrospective Analysis. Value in Health. 2010;13(6):853-856.

31. Grosse SD. Assessing cost-effectiveness in healthcare: history of the $\$ 50,000$ per QALY threshold. 2008.

32. Braithwaite RS, Meltzer DO, King JT, Jr., Leslie D, Roberts MS. What does the value of modern medicine say about the $\$ 50,000$ per quality-adjusted life-year decision rule? Medical care. 2008;46(4):349356.

33. World Health O. WHO-CHOICE (CHOosing Interventions that are Cost-Effective): Cost effectiveness and strategic planning, . In:2014.

34. Asim O, Petrou S. Valuing a QALY: review of current controversies. Expert review of pharmacoeconomics \& outcomes research. 2005;5(6):667-669.

35. Beresniak A, Medina-Lara A, Auray JP, et al. Validation of the Underlying Assumptions of the Quality-Adjusted Life-Years Outcome: Results from the ECHOUTCOME European Project. PharmacoEconomics. 2015;33(1):61-69.

36. Nuijten MJ, Dubois DJ. Cost-utility analysis: current methodological issues and future perspectives. Frontiers in pharmacology. 2011;2:29.

37. Ashcroft RE. Quality of Life as the Basis of Health Care Resource Allocation: A Philosopher's Perspective on QALYs. Virtual Mentor. 2005;7(2).

38. Department of Health MP, Industry G. A new valuebased approach to the pricing of branded medicines: a consultation. London: UK Secretary of State for Health Department of Health;2010.

39. German Institute for Q, Efficiency in Health C. General methods for the assessment of the relation of benefits to cost. Cologne: The Institute for Quality and Efficiency in Health Care;2009.

40. Bach PB, Pearson SD. Payer and policy maker steps to support value-based pricing for drugs. Jama. 2015;314(23):2503-2504.

41. Ocana A, Amir E, Tannock IF. Toward value-based pricing to boost cancer research and innovation. Cancer research. 2016;76(11):3127-3129.

42. Kaltenboeck A, Bach PB. Value-based pricing for drugs: theme and variations. Jama. 2018;319(21):2165-2166.

43. National Institute for $\mathrm{H}$, Care E. Value Based Assessment of Health Technologies. Centre for Health Technology Evaluation;2014.

44. Towse A. Value based pricing, research and development, and patient access schemes. Will the United Kingdom get it right or wrong? British journal of clinical pharmacology. 2010;70(3):360-366.
45. Lee TT, Gluck AR, Curfman G. The politics of Medicare and drug-price negotiation (updated). HEALTH AFFAIRS BLOG, Oct. 2016;20.

46. Davis MH. Should Medicare be Allowed to Negotiate Drug Prices? CQ Researcher. 2016;26(20):473.

47. Danzon P, Towse A, Mestre-Ferrandiz J. Value-Based Differential Pricing: Efficient Prices for Drugs in a Global Context. Health Economics. 2015;24(3):294301.

48. Institute for C, Economic R. Overview of the ICER value assessment framework and update for 20172019 In. Vol 20172017.

49. Institute for C, Economic R. Value Assesment Framework. In:2015.

50. Schnipper LE, Davidson NE, Wollins DS, et al. American Society of Clinical Oncology Statement: A Conceptual Framework to Assess the Value of Cancer Treatment Options. Journal of clinical oncology : official journal of the American Society of Clinical Oncology. 2015;33(23):2563-2577.

51. National Comprehensive Cancer N. NCCN Clinical Practice Guidelines in Oncology with NCCN Evidence Blocks.

https://www.nccn.org/professionals/physician_gls/d efault.aspx. Accessed January 18, 2019.

52. Memorial Sloan K. Drug Abacus. https://drugpricinglab.org/tools/drug-abacus/

53. Polite B, Conti RM, Ward JC. Reform of the Buy-andBill System for Outpatient Chemotherapy Care Is Inevitable: Perspectives from an Economist, a Realpolitik, and an Oncologist. American Society of Clinical Oncology educational book / ASCOAmerican Society of Clinical OncologyMeeting. 2015:e75-80.

54. Wonder M. Patient access to new cancer drugs in the United States and Australia. Value in Health. 2012;15(2):397-398.

55. Food US, Drug A. Fast Track, Breakthrough Theraphy, Accelerated Approval, Priority Review.

https://www.fda.gov/patients/learn-about-drug-anddevice-approvals/fast-track-breakthrough-therapyaccelerated-approval-priority-review. Published February 23, 2018. Accessed January 1, 2019

56. Singer DS, Jacks T, Jaffee E. A US "Cancer Moonshot" to accelerate cancer research. Science. 2016;353(6304):1105-1106.

57. Dangi-Garimella S. Patient Access to ImmunoOncology Agents - A US Policy Perspective. The American Journal of Managed Care. 2016;22(2):1719.

58. Drummond MF, McGuire A. Economic evaluation in health care: merging theory with practice. Oxford University Press, USA; 2001.

59. Corbacho B, Pinto-Prades JL. Health economic decision-making: a comparison between UK and Spain. British medical bulletin. 2012;103(1):5-20. 
60. Thomas D, John D, Ashoush N, Lega F, Li H. Market Access, Pharmaceutical Pricing, and Healthcare Costs. In: Clinical Pharmacy Education, Practice and Research. Elsevier; 2019:249-259.

61. Araújo FC, Gonçalves J, Fonseca JE. Pharmacoeconomics of biosimilars: what is there to gain from them? Current rheumatology reports. 2016;18(8):50.

62. Barzey V, Atkins MB, Garrison LP, Asukai Y, Kotapati S, Penrod JR. Ipilimumab in 2nd line treatment of patients with advanced melanoma: a costeffectiveness analysis. Journal of medical economics. 2012;16(2):202-212.

63. Chen TT. Milestone Survival: A Potential Intermediate Endpoint for Immune Checkpoint Inhibitors. Journal of the National Cancer Institute. 2015;107(9):10.1093/jnci/djv1156.

64. Wolchok JD, Hoos A, O'Day S, et al. Guidelines for the evaluation of immune therapy activity in solid tumors: immune-related response criteria. Clinical cancer research : an official journal of the American Association for Cancer Research. 2009;15(23):74127420.

65. Nishino M, Gargano M, Suda M, Ramaiya NH, Hodi FS. Optimizing immune-related tumor response assessment: does reducing the number of lesions impact response assessment in melanoma patients treated with ipilimumab. J Immunother Cancer. 2014;2:17.

66. Hodi FS, O'Day SJ, McDermott DF, et al. Improved survival with ipilimumab in patients with metastatic melanoma. New England Journal of Medicine. 2010;363(8):711-723.

67. Kantoff PW, Higano CS, Shore ND, et al. Sipuleucel-T immunotherapy for castration-resistant prostate cancer. New England Journal of Medicine. 2010;363(5):411-422.
68. Wolchok JD, Neyns B, Linette G, et al. Ipilimumab monotherapy in patients with pretreated advanced melanoma: a randomised, double-blind, multicentre, phase 2, dose-ranging study. The lancet oncology. 2010;11(2):155-164.

69. Maio M, Bondarenko I, Robert C, et al. Survival analysis with 5 years of follow-up in a phase III study of ipilimumab and dacarbazine in metastatic melanoma. Paper presented at: EUROPEAN JOURNAL OF CANCER; 2013.

70. Wolchok JD, Weber JS, Maio M, et al. Four-year survival rates for patients with metastatic melanoma who received ipilimumab in phase II clinical trials. Annals of Oncology : Official Journal of the European Society for Medical Oncology / ESMO. 2013;24(8):2174-2180.

71. Fine GD. Consequences of delayed treatment effects on analysis of time-to-event endpoints. Drug information journal. 2007;41(4):535-539.

72. Chen T-T. Statistical issues and challenges in immunooncology. J Immuno Ther Cancer. 2013;1:18.

73. Annemans L, Asukai Y, Barzey V, et al. MO2 Extrapolation in oncology modelling: novel methods for novel compounds. Value in Health. 2011;14(7):A242-A243.

74. Akaike H. Information Theory and an Extension of the Maximum Likelihood Principle. In: Petrov BN, Csaki F, eds. Second International Symposium on Information Theory. Budapest: Academiai Kiado; 1973:267.

75. Austin PC. An introduction to propensity score methods for reducing the effects of confounding in observational studies. Multivariate behavioral research. 2011;46(3):399-424.

76. Imbens GM, Wooldridge JM. Recent developments in the econometrics of program evaluation. Journal of economic literature. 2009; 47(1):5-86 